\title{
Article \\ The Analysis of Periodic Order in Monolayers of Colloidal Superballs
}

\author{
Daniël N. ten Napel ${ }^{1}\left(\mathbb{D}\right.$, Janne-Mieke Meijer ${ }^{2}(\mathbb{D})$ and Andrei V. Petukhov $1,3, * \mathbb{D}$ \\ 1 Van 't Hoff Laboratory for Physical and Colloid Chemistry, Debye Institute for Nanomaterials Science, \\ Utrecht University, Padualaan 8, 3584 CH Utrecht, The Netherlands; d.n.tennapel@uu.nl \\ 2 Soft Matter and Biological Physics, Department of Applied Physics, Eindhoven University of Technology, \\ P.O. Box 513, 5600 MB Eindhoven, The Netherlands; j.m.meijer@tue.nl \\ 3 Laboratory of Physical Chemistry, Department of Chemical Engineering and Chemistry, \\ Eindhoven University of Technology, P.O. Box 513, 5600 MB Eindhoven, The Netherlands \\ * Correspondence: a.v.petukhov@uu.nl; Tel.: +31-30-253-1167
}

Citation: ten Napel, D.N.; Meijer, J.-M.; Petukhov, A.V. The Analysis of Periodic Order in Monolayers of Colloidal Superballs. Appl. Sci. 2021, 11, 5117. https://doi.org/ 10.3390/app11115117

Academic Editor: Sebastian Jaksch

Received: 10 May 2021

Accepted: 26 May 2021

Published: 31 May 2021

Publisher's Note: MDPI stays neutral with regard to jurisdictional claims in published maps and institutional affiliations.

Copyright: () 2020 by the authors. Licensee MDPI, Basel, Switzerland. This article is an open access article distributed under the terms and conditions of the Creative Commons Attribution (CC BY) license (https:// creativecommons.org/licenses/by/ $4.0 /)$.

\begin{abstract}
The characterization of periodic order in assemblies of colloidal particles can be complicated by the coincidence of Bragg diffraction peaks of the structure and minima in the form factor of the particles. Here, we demonstrate a general strategy to overcome this problem that is applicable to all low-dimensional structures. This approach is demonstrated in the application of small-angle X-ray scattering (SAXS) for the characterization of monolayers of colloidal silica superballs prepared using the unidirectional rubbing method. In this method, the ordering of the colloidal superballs is achieved by mechanically rubbing them onto a polydimethylsiloxane (PDMS)-coated surface. Using three differently shaped superballs, ranging from spherical to almost cubic, we show that certain Bragg peaks may not appear in the diffraction patterns due to the presence of minima in the form factor. We show that these missing Bragg peaks can be visualized by imaging the colloidal monolayers at various orientations. Moreover, we argue that the same strategy can be applied to other techniques, such as neutron scattering.
\end{abstract}

Keywords: colloidal superballs; colloidal monolayers; SAXS; scattering; form factor

\section{Introduction}

Colloidal monolayers are used in a wide variety of applications, such as anti-reflective coatings, photonic materials, photovoltaics and biosensors [1,2]. Alternatively, the assembly of colloids into monolayers is often used as a step in colloidal syntheses in order to locally modify the particles in order, for example, to produce Janus colloids [3-5]. Various methods are known for the preparation of colloidal monolayers, such as drying methods, spin coating, the assembly on liquid interfaces and the horizontal and vertical deposition methods [2]. Each of these preparation methods have their own strengths and weaknesses. It was only recently shown, however, that colloidal monolayers could also be prepared by mechanically rubbing dried colloids between two sticky surfaces such as polydimethylsiloxane (PDMS) [6,7]. This quick, simple and inexpensive method produces high-quality colloidal monolayers where the colloidal crystal grains align in the direction in which the mechanical rubbing was applied.

So far, the unidirectional rubbing method has only been used for the monolayer formation of spherical particles. However, the shape of the colloidal particles can greatly influence the structures that are formed [8]. By employing the unidirectional rubbing method for the assembly of anisotropic colloids, colloidal monolayers that possess unique properties may be formed, which might be of interest in one of the many colloidal monolayer applications. Of specific interest are superball-shaped colloids, i.e., shapes varying from spheres via cubes with rounded edges to perfect cubes, because their variable shape allows smooth phase transitions to occur [9]. For instance, upon allowing superball-shaped colloids to 
sediment, plastic crystals and solid-solid phase transitions were observed [10,11], whereas upon assembling these particles into monolayers using the horizontal and vertical deposition method both predicted densest packings were found as well as a continuous transition between the two lattice structures [12,13]. Furthermore, upon the addition of depletants, the resulting crystal phases were found to depend on both the shape of the superballs and the radius of gyration of the added depletant relative to the size of the superballs [9,14]. By employing the unidirectional rubbing method for the organization of colloidal superballs into monolayers it can be expected that monolayers with unique organizations can be achieved as a result of the directional force applied during its assembly.

The periodic structures in colloidal monolayers can be studied, on the one hand, using microscopy techniques, which are superior in revealing local densely packed structures. On the other hand, to characterize long-range ordering on the colloidal scale, small-angle neutron scattering (SANS) [15] and small-angle X-ray scattering (SAXS) [16] are often applied. Although these two techniques are very similar in their general approach and theoretical models, there are many factors that determine which of these scattering techniques is most suitable for a certain study. For example, while for $\mathrm{X}$-rays the scattering contrast is essentially determined by the total local electron density, which is approximately proportional to the mass density, neutron scattering contrast has much higher sensitivity to most lighter atoms with fewer electrons. Moreover, SANS offers great opportunities for contrast variation by using isotopes, most remarkably hydrogen/deuterium. This allows the revelation of minor structural details [17] or the minimization of multiple scattering [18]. In addition, the scattering of spin-polarized neutrons is sensitive to the magnetic ordering in the sample [19]. Wide opportunities can be opened by combining SANS with advanced spin-echo techniques, such as spin-echo small-angle neutron scattering (SESANS), which can access large-period structures [20] as well as nanostructures along with their nanosecond temporal dynamics [21,22]. The recent developments in synchrotron sources, $\mathrm{X}$-ray detectors, and X-ray optics paved the way to millisecond SAXS data acquisition, microradian angular resolution, the possibility to scan samples with a submicrometer-sized beam and the application of coherent X-ray scattering techniques $[23,24]$. Due to all these achievements, contemporary SAXS and SANS became truly complementary techniques.

In this work we have studied the ordered monolayer structures of colloidal superballs similar in size but with different shapes, ranging from spherical to almost cubic, prepared using the unidirectional rubbing method. Since these structures are static, the contrast is high, and because high angular resolution is required due to the large structure period, SAXS is the technique of choice in the present work. The small-angle scattering from a periodic arrangement of particles does not only depend on their mutual spatial arrangement, but also on the shape of the individual particles. The latter is described by the form factor. For spherically symmetric particles the form factor is isotropic because the X-rays are scattered equally in all directions and the form factor can be calculated analytically. For non-spherical particle shapes, however, the form factor is no longer isotropic, which complicates the analysis of the scattering patterns. It was previously shown by Meijer et al. that the form factor of hollow silica superballs with a thin shell and distinct cubic shape become cross-like with little intensity in the corners of the diffraction patterns [11]. These effects in the form factor will result in Bragg peaks in these locations to be less visible, which complicates the structural analysis. As we faced in the present study, the presence of minima in the form factor of hollow superball particles can make many important diffraction peaks invisible, which made the structure characterization complicated if not impossible.

Here, we present a strategy for the measurement and analysis of the SAXS diffraction patterns of monolayers of colloidal silica superballs prepared using the unidirectional rubbing method. We show that by performing the measurements under a range of rotations, a detailed analysis can be performed of both the form factor and structure factor. In this case the $q$-values of the Bragg peaks can be shifted away from the minima in the form factor. The effect of sample rotation is first illustrated using spheres for which the form factor is isotropic and can be calculated analytically. We then show for the more cubic 
superballs, for which the form factor cannot be calculated analytically, that this rotation allows us to visualize and assign all Bragg diffraction peaks for a full structural analysis. Finally, we present a model for the phase transition observed in the lattices of colloidal superball monolayers prepared using the unidirectional rubbing method upon varying the shape of the colloidal superballs from spherical to almost cubic.

\section{Theory}

In SAXS the scattered intensity is the product of the form factor and the structure factor:

$$
I(\vec{q})=P(\vec{q}) S(\vec{q}),
$$

where $\vec{q}$ is the scattering vector. The form factor $P(\vec{q})$ is determined by the shape and size of the individual particles. For uniform spherical particles the form factor is radially symmetric and equal to:

$$
P(\vec{q})=P(q)=\frac{3[\sin (q R)-(q R) \cos (q R)]}{(q R)^{3}},
$$

where $q$ is the length of the scattering vector and $R$ is the radius of the spherical particle. Due to the symmetry of the spherical shape, the form factor does not depend on the particle orientation. For non-spherical particles, however, the form factor does depend on the orientation of the particle. While most anisotropic shapes do not have an analytical solution, their form factor can always be calculated numerically.

The structure factor $S(\vec{q})$, on the other hand, is determined by the structural assembly of the particles. Consider an ideal two-dimensional lattice with lattice points at distance vectors of:

$$
\vec{r}=m \vec{a}_{1}+n \vec{a}_{2},
$$

where $m$ and $n$ are integers and $\vec{a}_{1}$ and $\vec{a}_{2}$ are the primitive lattice vectors. The structure factor of any structural assembly is equal to:

$$
S(\vec{q})=\frac{1}{N}\left|\sum_{n=1}^{N} e^{i \vec{q} \cdot \vec{r}_{n}}\right|^{2},
$$

where $N$ is the total number of particles and $\vec{r}_{n}$ is the distance vector of the $n$th particle. For an ideal two-dimensional lattice the structure factor is non-zero at:

$$
\vec{q}=h \vec{b}_{1}+k \vec{b}_{2}
$$

for any value of $q_{z}$. Here, $h$ and $k$ are the Miller indices, $\vec{b}_{1}$ and $\vec{b}_{2}$ are the primitive reciprocal basis vectors and $q_{z}$ is the component of the scattering vector that is parallel to the incoming $\mathrm{X}$-ray beam. The angle between these primitive reciprocal basis vectors is defined as $\beta$. The primitive reciprocal basis vectors are related to the primitive lattice vectors as follows:

$$
\vec{b}_{1}=2 \pi \frac{\vec{a}_{2} \times \vec{n}}{\left|\vec{a}_{1} \times \vec{a}_{2}\right|}, \vec{b}_{2}=2 \pi \frac{\vec{n} \times \vec{a}_{1}}{\left|\vec{a}_{1} \times \vec{a}_{2}\right|},
$$

where $\vec{n}$ is a vector normal to the two-dimensional lattice plane. Finally, the angle between the primitive lattice vectors is $\alpha=180^{\circ}-\beta$.

\section{Materials and Methods}

\subsection{Preparation of the Colloidal Superball Monolayers}

The solid colloidal silica spheres were synthesized using the Stöber process [25]. Two types of cube-like hollow colloidal superballs were synthesized by coating precursor colloidal hematite cubes with a layer of silica and subsequently dissolving the magnetic core particle $[9,26]$. The superball family describes the shape that smoothly interpolates 
between a sphere and a cube. The contours of the superball shape are defined by the following formula [13]:

$$
\left|\frac{2 x}{D}\right|^{m}+\left|\frac{2 y}{D}\right|^{m}+\left|\frac{2 z}{D}\right|^{m}=1
$$

where $m$ is the shape parameter, $D$ the diameter (face-to-face length) of the superballs and $x, y$ and $z$ are the coordinates of the surface along the $x-, y$ - and $z$-axes, respectively. The average shape parameter $m$ and diameter $D$ of the synthesized superball colloids were determined from transmission electron microscopy (TEM) images using a customwritten analysis script written in Python. The resulting spherical, semi-cubic and cube-like particles were found to possess average shape parameters of $m=2,2.87$ and 3.63 and diameters of $D=1.10,0.96$ and $1.01 \mu \mathrm{m}$, respectively. These colloidal silica superballs were assembled into monolayers by mechanically rubbing them onto polydimethylsiloxane (PDMS)-coated glass slides (\#0, $60 \mathrm{~mm} \times 24 \mathrm{~mm} \times 0.085-0.12 \mathrm{~mm}$ ) using a custom-made device. For all samples, this device was set to run 10 rubbing cycles consisting of a back and forth motion of a PDMS cylinder with a diameter of $1 \mathrm{~cm}$ at a rubbing pressure of $200 \mathrm{~g}$ and a rubbing speed of $3 \mathrm{~mm} / \mathrm{s}$. At the end of each rubbing motion, the PDMS cylinder was lifted over the remaining colloidal powder. Finally, the excess colloidal powder was removed using a strong nitrogen flow. TEM images of the individual colloidal superballs and scanning electron microscopy (SEM) images of their corresponding monolayers are depicted in Figure 1a-f.
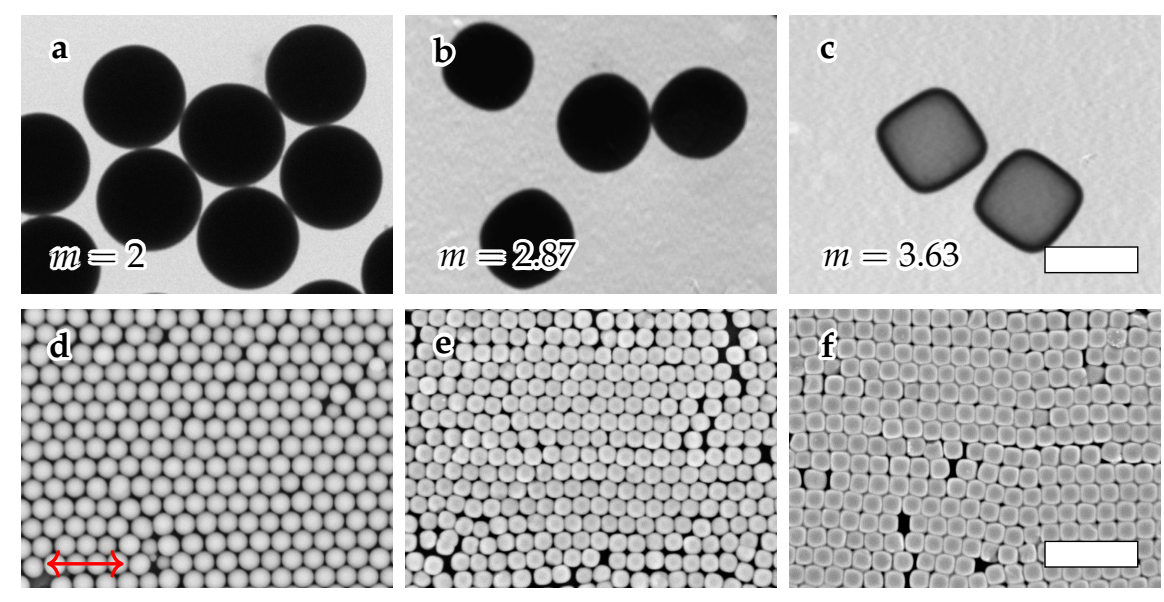

Figure 1. Representative (a-c) transmission electron microscopy (TEM) images of the colloidal superballs and (d-f) scanning electron microscopy (SEM) images of their corresponding monolayers assembled using the unidirectional rubbing method. The direction of the mechanical rubbing is indicated in the images using a red arrow. The scale bars represent $(\mathbf{a}-\mathbf{c}) 1$ and $(\mathbf{d}-\mathbf{f}) 5 \mu \mathrm{m}$.

\subsection{Analysis of the Colloidal Superball Monolayers Using SAXS}

The SAXS measurements were performed at the BM26B DUBBLE beamline of the ESRF in Grenoble, France using the microradian setup [23] employing compound refractive lenses [27]. The X-ray beam with a photon energy of $12 \mathrm{kV}$ was focused on a CCD with a resolution of $4008 \times 2671$ pixels and pixel size of $22 \times 22 \mu \mathrm{m}$ (Photonic Science) placed at a distance of $7.5 \mathrm{~m}$ from the sample. A wedge-shaped beam-stop was used in order to prevent damage to the detector that can result from an exposure to the direct $X$-ray beam. The colloidal monolayers were placed perpendicular to the $\mathrm{X}$-ray beam with the rubbing direction along to the horizontal plane ( $x$-axis). The samples were rotated around the $x$-axis from $\phi=-60$ to $60^{\circ}$. The SAXS setup is schematically depicted in Figure 2. 


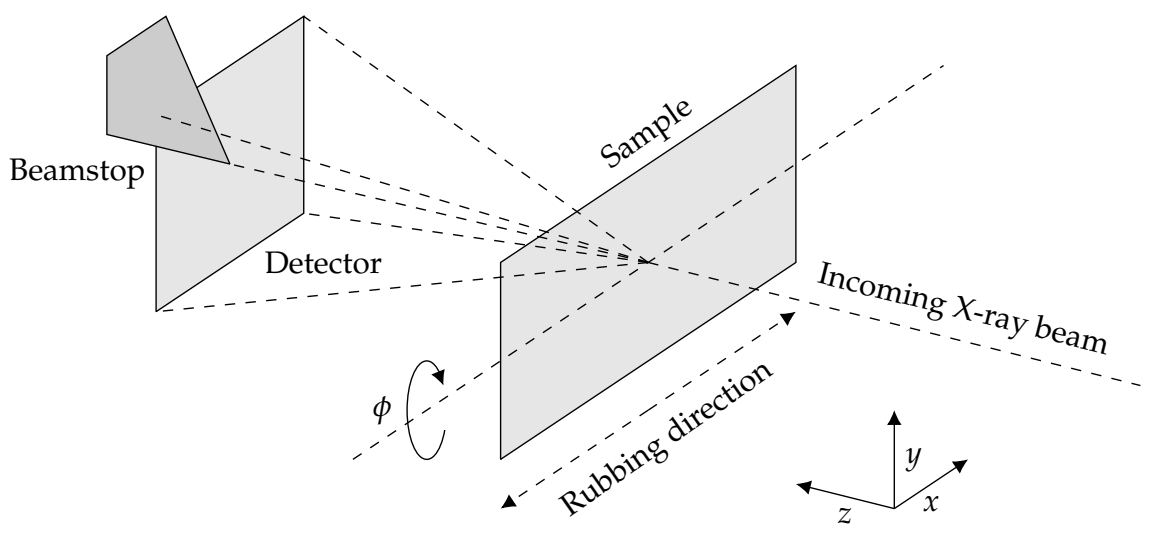

Figure 2. A schematic representation of the small-angle X-ray scattering (SAXS) setup. Here, the monolayers of colloidal superballs are rotated around the $x$-axis. The angle of rotation around this axis is denoted by $\phi$. The incoming X-ray beam travels along the $z$-axis. The scattering patterns are imaged in $x, y$-space. A beam-stop was used to prevent damage to the detector. Note that this schematic representation is not drawn to scale.

2D SAXS patterns were obtained at different rotations of $\phi$ with an exposure time of $20 \mathrm{~s}$. Dark current and background corrections were performed on the patterns before their analysis. The background images were obtained from a blank PDMS-coated glass slide at an equal rotation of $\phi$. Every measured diffraction pattern image was subtracted with the background imaged at an equal rotation in the $x$-axis to correctly remove the scattering background that is caused by the PDMS-coated glass slide. Custom analysis scripts written in Python were used to determine the positions of the peaks in the cross sections of the diffraction pattern images and to numerically calculate the form factor of the different superball shapes. To model the form factor, we constructed the superballs, whether solid or hollow, on a three-dimensional raster with 30 points along each dimension. The interference between the points within the particles' shape was then calculated and converted via a Fourier transform.

\section{Results and Discussion}

To elucidate the role of the particles' form factor on the structural analysis in (X-ray) diffraction patterns, monolayers of superball colloids with three different shapes, namely spherical, semi-cubic and cube-like, were prepared using the unidirectional rubbing method and investigated with SAXS. These colloidal monolayers were rotated around the rubbing direction ( $x$-axis). First, we focus on 2D SAXS patterns and their analysis for a monolayer obtained from spherical colloids that can be described as a superball with a shape parameter of $m=2$. Figures $3 \mathrm{a}-\mathrm{c}$ show the SAXS patterns obtained for a monolayer prepared from solid colloidal silica spheres with $D=1.10 \mu \mathrm{m} \pm 3.2 \%$. The three typical diffraction patterns imaged at rotations of $\phi=0,20$ and $40^{\circ}$ all show distinct peaks that indicate the spheres have formed well-defined periodic structures in the monolayer. The presence of separate peaks, instead of a series of co-axial rings, indicate that the crystal orientation within the imaged area of the colloidal monolayer is fixed. This is a known result of the unidirectional rubbing method where the crystal grains align in the direction of rubbing $[6,7]$.

Figure 3a shows the diffraction pattern imaged at a rotation of $\phi=0^{\circ}$, where six-fold Bragg diffraction peaks indicate that the crystal structure of the colloidal monolayer is a two-dimensional close-packed hexagonal lattice. The peaks centered along the vertical direction of the images can be identified as the $h k=10$ and 20 peaks of a hexagonal lattice, as indicated in Figure 3a. Upon rotating the sample around the $x$-axis, the Bragg peaks move in the vertical $y$-direction, which makes the diffraction pattern appear elongated (Figure $3 b, c)$. In addition, it can be seen that the intensities of the peaks in the diffraction patterns change relative to each other. Specifically, the 10 peak is strongly visible in the pattern at $\phi=0$ and $20^{\circ}$, but disappears at $\phi=40^{\circ}$, while the 20 peak is visible at $\phi=0$ 
and $40^{\circ}$, but disappears at $\phi=20^{\circ}$. By contrast, the 30 peak is only slightly visible at $\phi=20^{\circ}$ and invisible at both $\phi=0$ and $40^{\circ}$. These trends can be more easily visualized using the cross sections of the scattering patterns in the vertical direction at $q_{x}=0 \mu \mathrm{m}$. Our analysis revealed that upon rotating the sample the positions of the maxima in the structures shifted to a higher $q_{y}$-values. This shift is directly related to the rotation and by scaling the scattering vector $q_{y}$ with a factor of $\cos (\phi)$ all peaks in the various cross sections fell at equal values of $q_{y} \cos (\phi)$, as shown in Figure $3 \mathrm{~d}$ for $\phi=0,10,20,30$ and $40^{\circ}$. In this case we find maxima at $q_{y} \cos (\phi)=5.95,11.90$ and $17.85 \mu^{-1}$ for the 10,20 and 30 peaks, respectively. Thus, by considering the cross sections of the diffraction patterns imaged at various rotations of $\phi$, a more precise analysis can be performed to locate the positions of the various $h k$ peaks.
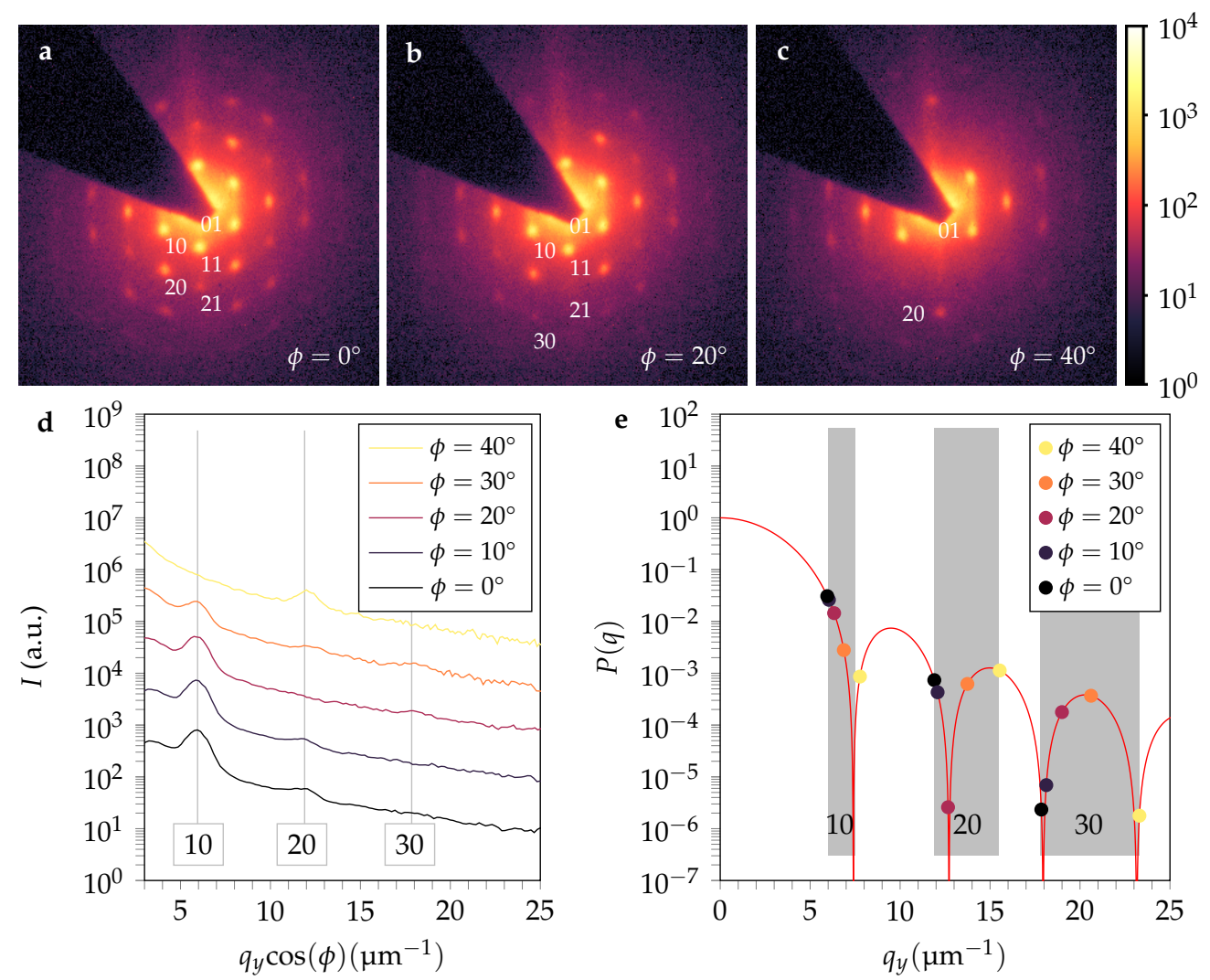

Figure 3. $(\mathbf{a}-\mathbf{c})$ 2D SAXS patterns of a monolayer prepared from colloidal silica spheres with an average diameter of $D=1.10 \mu \mathrm{m} \pm 3.2 \%$ imaged at rotations around the $x$-axis of $\phi$. The Bragg peaks are labelled on their left. (d) Cross sections of the diffraction patterns at different $\phi$ taken at $q_{x}=0 \mu \mathrm{m}^{-1}$ versus $q_{y} \cos (\phi)$, revealing that the positions of the identified $h k$ peaks occur at specific $q_{y} \cos (\phi)$ positions. (e) Positions of the 10, 20 and 30 peaks for different $\phi$ overlayed on top of the calculated spherical form factor $P(q)$.

The strong changes in the intensity of the peaks observed upon rotating the sample are due to the presence of minima in the form factor. The distances of periodicity in the sample in the $y$-direction decreased upon rotation and thus correspond to higher $q_{y}$-values in reciprocal space. Figure 3e shows this process for the positions of the 10, 20 and 30 peaks at different $\phi$ plotted on top of the calculated spherical form factor $P(q)$. It can be seen that the positions of the $h k$ peaks shifted to higher $q_{y}$-values with increasing $\phi$, thus resulting in changes in their relative intensities. This example shows that if a large enough range in the scattering vector $q_{y}$ can be probed to shift the positions of these $h k$ peaks away from the $P(q)$ minima, all peaks can be identified correctly. Similar analyses can be performed to determine the positions of the other $h k$ peaks present in the scattering pattern. The 01 peak, 
for example, is visible in each of the diffraction patterns depicted in Figure 3a-c, whereas both the 11 and 21 peaks are only visible up to a rotation of $\phi=20^{\circ}$ (Figure 3a,b).

Next, we discuss the diffraction patterns of a monolayer prepared from hollow colloidal silica superballs with $m=2.87 \pm 13 \%$ and $D=0.96 \mu \mathrm{m} \pm 4.5 \%$ that have a semicubic shape and, in addition, are now hollow shells, which will influence the form factor. Figure $4 \mathrm{a}-\mathrm{c}$ show the 2D SAXS patterns for different rotations $\phi$. Here, distinct Bragg peaks can again be observed, which we can identify as the different $h k$ peaks as indicated in Figure 4a-c. Similar to the spherical colloids, we observed that different peaks are visible at different rotations. At $\phi=0^{\circ}$, the 10 peak is only barely visible while both the 20 and 30 peaks are clearly visible. Interestingly, and perhaps coincidentally, at $\phi=20^{\circ}$ no peaks are visible all along the vertical axis at $q_{x}=0 \mu \mathrm{m}^{-1}$. At $\phi=40^{\circ}$, the 10 and 20 peaks appear again while the 30 peak is still invisible.
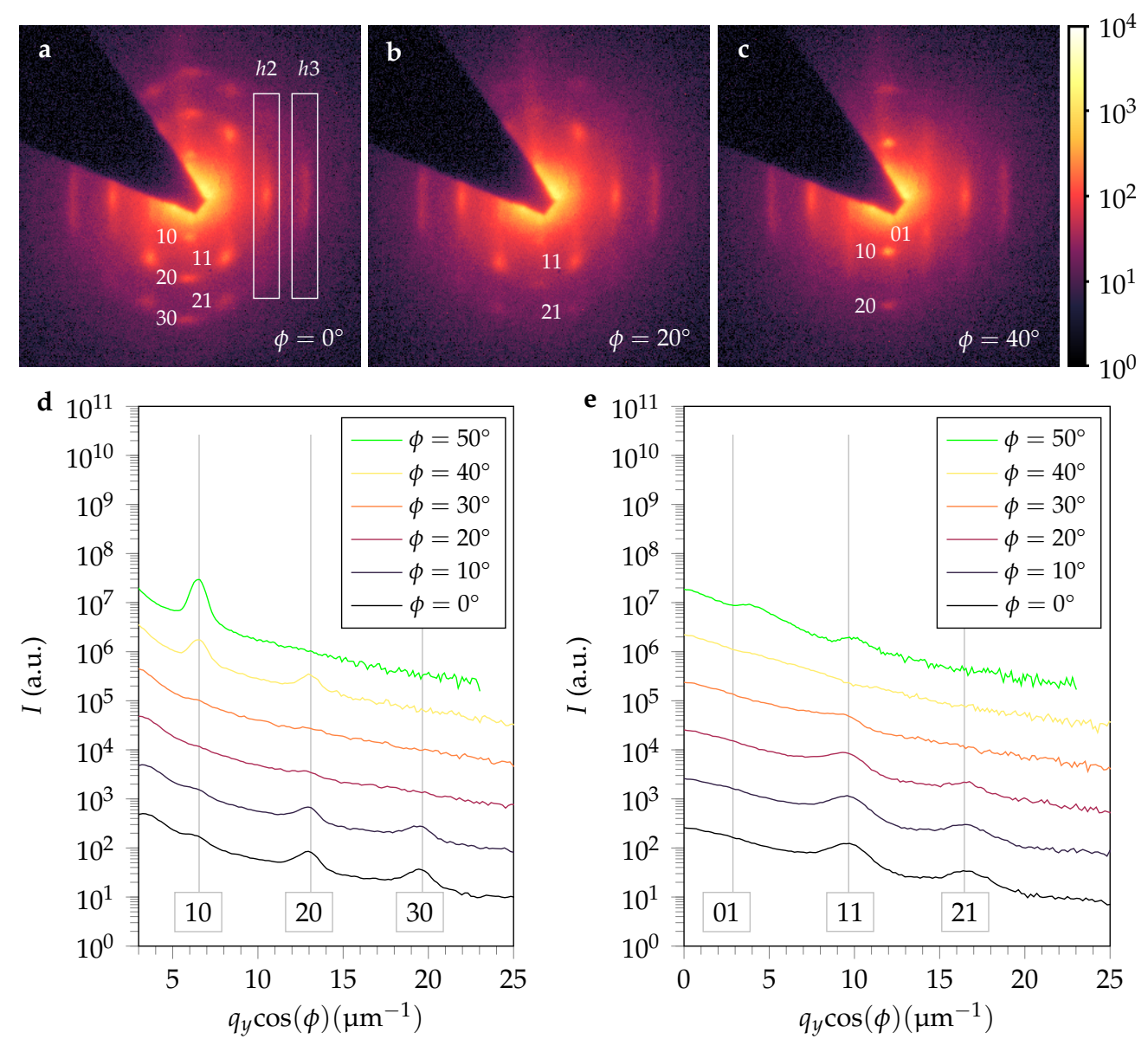

Figure 4. $(\mathbf{a}-\mathbf{c})$ 2D SAXS patterns of a monolayer prepared from hollow colloidal silica superballs with an average shape parameter of $m=2.87 \pm 13 \%$ and diameter of $D=0.96 \mu \mathrm{m} \pm 4.5 \%$ imaged at different rotations around the $x$-axis of $\phi$. The Bragg peaks are labelled on their left. (d-e) Vertical cross sections of the diffraction patterns at (d) $q_{x}=0 \mu \mathrm{m}^{-1}$ and (e) $q_{x}=6.12 \mu \mathrm{m}^{-1}$. The vertical lines indicate the $q_{y} \cos (\phi)$ positions of the identified peaks.

Figure $4 \mathrm{~d}$ shows the cross sections taken along the vertical axis at $q_{x}=0 \mu \mathrm{m}^{-1}$. Again, in these cross sections the observed peak behavior can be more clearly visualized. The 10 peak is only visible at high $\phi$ values, while the 30 is only visible at low $\phi$ values and no peaks are observed at rotations in the $x$-axis of $\phi=20$ and $30^{\circ}$. The appearance and disappearance of the peaks in the scattering patterns upon rotation for these superball monolayers is distinctly different from the behavior observed for the monolayers consisting of spheres. The explanation of this behavior is that the particle form factor, now from a hollow and anisotropic particle, clearly influenced the appearance of structure factor peaks 
considerably, as expected. To further characterize and understand these non-trivial SAXS patterns, it can be useful to also consider vertical cross sections at different $q_{x}$ positions. Figure $4 \mathrm{e}$ depicts the vertical cross sections taken at $q_{x}=6.12 \mu \mathrm{m}^{-1}$, which contain the $h 1$ peaks. Here, it can be seen that there is a clear evolution of the peaks. Specifically, the 01 peak becomes visible only at $\phi=50^{\circ}$. By contrast, the 11 and 21 peaks are both visible at up to $\phi=20^{\circ}$ and disappear at higher angles with only the 11 peak appearing again at $\phi=50^{\circ}$. The observation that both the 10 and the 01 peaks only appear upon a considerable rotation indicates that these peaks must be close to a minimum in the form factor. Furthermore, it can be seen that the $h 2$ and $h 3$ peaks appear to be broadened along the vertical $y$-direction, as indicated in Figure $4 a-c$.

The biggest influence of the particle form factor was observed for monolayers prepared from hollow superballs with $m=3.63 \pm 12 \%$ and $D=1.01 \mu \mathrm{m} \pm 6.6 \%$ that have a distinct cube-like shape. Figure 5a-c show the 2D SAXS patterns imaged at different rotations. Strikingly, here not even a single Bragg peak is significantly visible in the pattern at a rotation of $\phi=0^{\circ}$ as seen in Figure 5a. Upon rotating the sample to $\phi=20$ and $40^{\circ}$, some of the diffraction peaks can be made visible (Figure $5 b, c$ ).
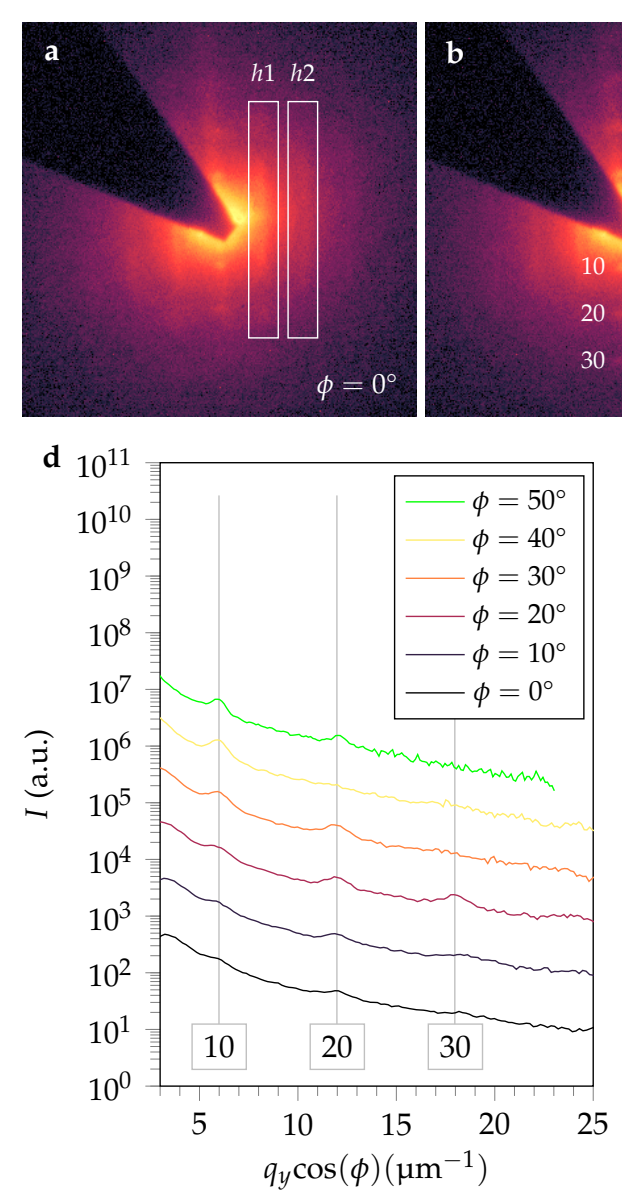
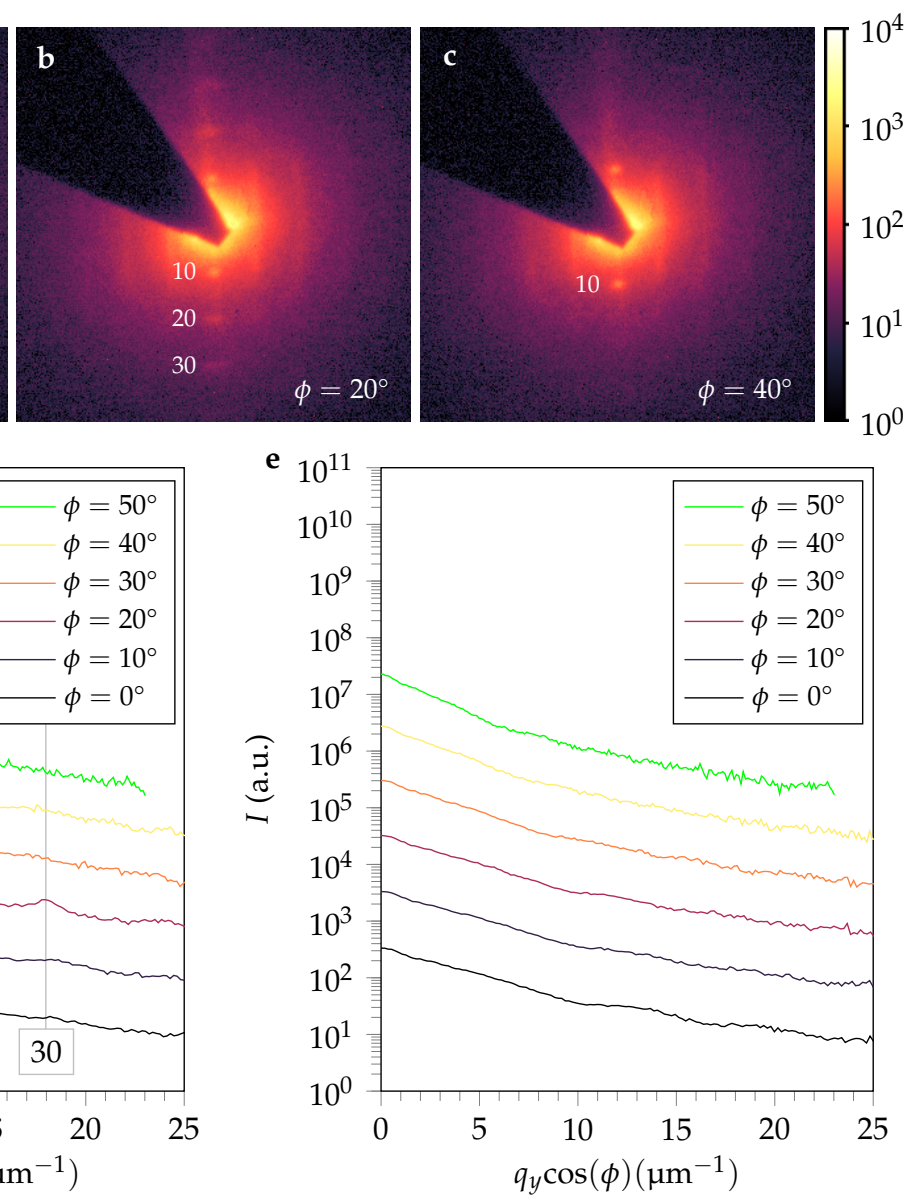

Figure 5. $(\mathbf{a}-\mathbf{c})$ 2D SAXS patterns of a monolayer prepared from hollow colloidal silica superballs with an average shape parameter of $m=3.63 \pm 12 \%$ and diameter of $D=1.01 \mu \mathrm{m} \pm 6.6 \%$ imaged at different rotations around the $x$-axis of $\phi$. The Bragg peaks are labelled on their left. (d,e) Vertical cross sections of the diffraction patterns at (d) $q_{x}=0 \mu \mathrm{m}^{-1}$ and (e) $q_{x}=5.76 \mu \mathrm{m}^{-1}$. The vertical lines indicate the $q_{y} \cos (\phi)$ positions of the identified peaks.

Again, we examine the vertical cross sections of the diffraction patterns at $q_{x}=0 \mu \mathrm{m}^{-1}$ (Figure 5d). From the evolution of the peaks, it is clear that the 10 peak appears in the patterns at $\phi=30^{\circ}$ and higher rotations, while the 20 and 30 peaks are only visible up to $\phi=30^{\circ}$. Furthermore, in the scattering patterns depicted in Figure $5 \mathrm{a}-\mathrm{c}$, some intensity can 
be seen where the $h 1$ and $h 2$ peaks are expected. These regions are highlighted in Figure $5 \mathrm{a}$. In Figure $5 \mathrm{e}$ the cross sections of the diffraction patterns imaged at the various rotations $\phi$ taken at $q_{x}=5.76 \mu^{-1}$ are depicted, corresponding to the intensity ascribed to the $h 1$ peaks. Due to their broadness, however, no clear Bragg peaks can be assigned here as the $h 1$ peaks appear to form a continuous band.

To further understand the appearance and disappearance of the peaks, we examine the different form factor contribution of the superball colloids used here, with spherical, semi-cubic and cube-like shapes. The form factor of a hollow superball is a function of the shape and size as well as the thickness of the shell for which there is no analytical solution. Therefore, we have calculated their form factors numerically. Figure 6a-c show the resulting calculated 2D form factors at a rotation of $\phi=0^{\circ}$, in which the semi-cubic and cube-like superballs lie flat on the PDMS-coated substrate, and an effectively 2D projection is obtained. Although the form factor for spherical particles has an analytical solution, as seen in Figure 3e, we included it here as a numerical calculation for a direct comparison. It can be seen that upon increasing the shape parameter $m$ of the colloidal superballs, the isotropic rings visible for spheres $(m=2)$ began to show oscillations with maxima in the horizontal and vertical directions, i.e., in the directions of the flat sides of hollow superballs, and minima in the diagonal directions, i.e., the corners of the hollow superballs. These changes in the form factor explain why the structure factor peaks visible for the spherical particles disappear for the hollow superballs.
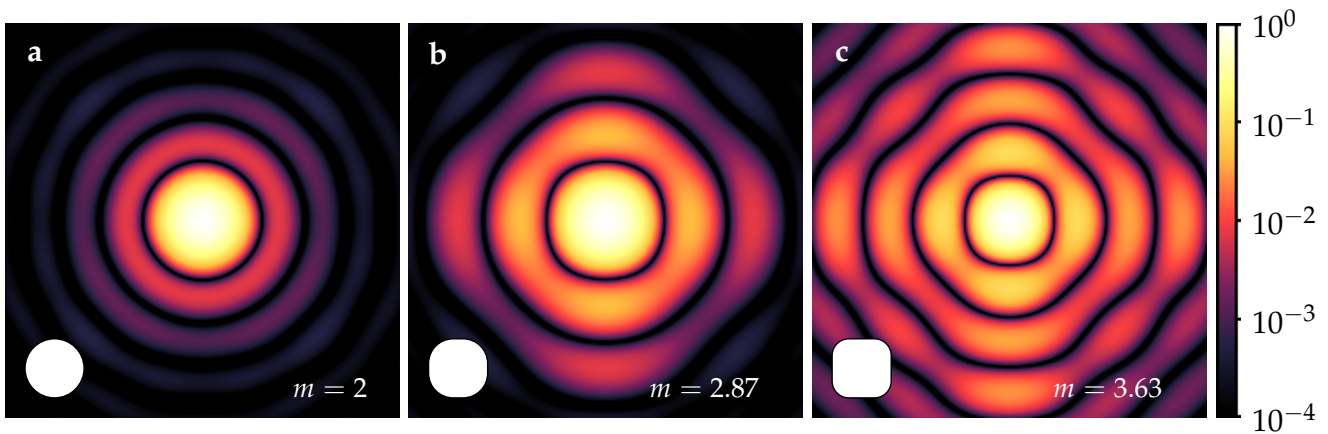

Figure 6. Numerically calculated form factors of the (a) solid spherical, (b) hollow semi-cubic and (c) hollow cube-like superballs at a rotation of $\phi=0^{\circ}$. The orientation of the superball particles is depicted in the insets of the images. Here, the intensity is limited to 4 orders of magnitude, which is approximately the same range the detector used in these experiments is able to measure.

Now that we have assigned all identifiable Bragg peaks in the diffraction patterns of the three superball shapes, we can begin to deduce their structure factors $S(\vec{q})$ and determine what these superball lattices look like in real space. From the positions of the 10, 20 and 30 peaks we can determine the length of the first primitive reciprocal basis vector, $\vec{b}_{1}$, according to Equation 5. For the spherical and semi-cubic superballs, we can determine the length of the second primitive reciprocal basis vector, $\vec{b}_{2}$, from the positions of the 01 , 11 and 21 peaks. Since for the cube-like superballs the $01,11,21$ peaks appear to form a continuous band, we can instead define $\vec{b}_{2 x}$ as the horizontal component of $\vec{b}_{2}$. Here, $\vec{b}_{2 x}$ is perpendicular to $\vec{b}_{1}$. The deduced structure factors for the spherical, semi-cubic and cube-like superballs along with the identified Bragg peaks and their primitive reciprocal basis vectors are depicted in Figure $7 \mathrm{a}-\mathrm{c}$, respectively. From the primitive reciprocal basis vectors $\vec{b}_{1}$ and $\vec{b}_{2}$, we can extract the real space primitive lattice vectors $\vec{a}_{1}$ and $\vec{a}_{2}$ and, in the case of the cubic-like superballs, $\vec{a}_{2 x}$. Their corresponding real space lattices are shown in Figure $7 d-f$. For the spherical superballs we determined the length of the primitive lattice vectors to be $a_{1}=1.21 \pm 0.013 \mu \mathrm{m}$ and $a_{2}=1.19 \pm 0.004 \mu \mathrm{m}$, with an angle of $\alpha=119.4 \pm 0.26^{\circ}$. This confirms that these superballs indeed form a hexagonal lattice in real space with a well-defined and equal periodicity in each direction. For the semi-cubic superballs we found that $a_{1}=1.11 \pm 0.019 \mu \mathrm{m}$ and $a_{2}=1.04 \pm 0.011 \mu \mathrm{m}$, separated by an 
angle of $\alpha=117.8 \pm 0.47^{\circ}$. Here, since $a_{1}$ is significantly larger than $a_{2}$, these semi-cubic superballs form a distorted hexagonal $\left(\Lambda_{0}\right)$ lattice as expected for the densest packing of superdisks in 2D [28]. From $a_{1}>a_{2}$ we further conclude that these semi-cubic superballs are oriented with their flat faces in the horizontal and vertical directions. For the cubiclike superballs that form a more cubic-like lattice we find that $a_{1}=1.05 \pm 0.027 \mu \mathrm{m}$ and $a_{2 x}=1.08 \pm 0.044 \mu \mathrm{m}$. Furthermore, from the presence of broad and continuous $h 1$ and $h 2$ bands in $S(\vec{q})$, we further conclude that these cube-like colloidal superballs form chains in the horizontal direction that are able to slide freely with respect to each other.
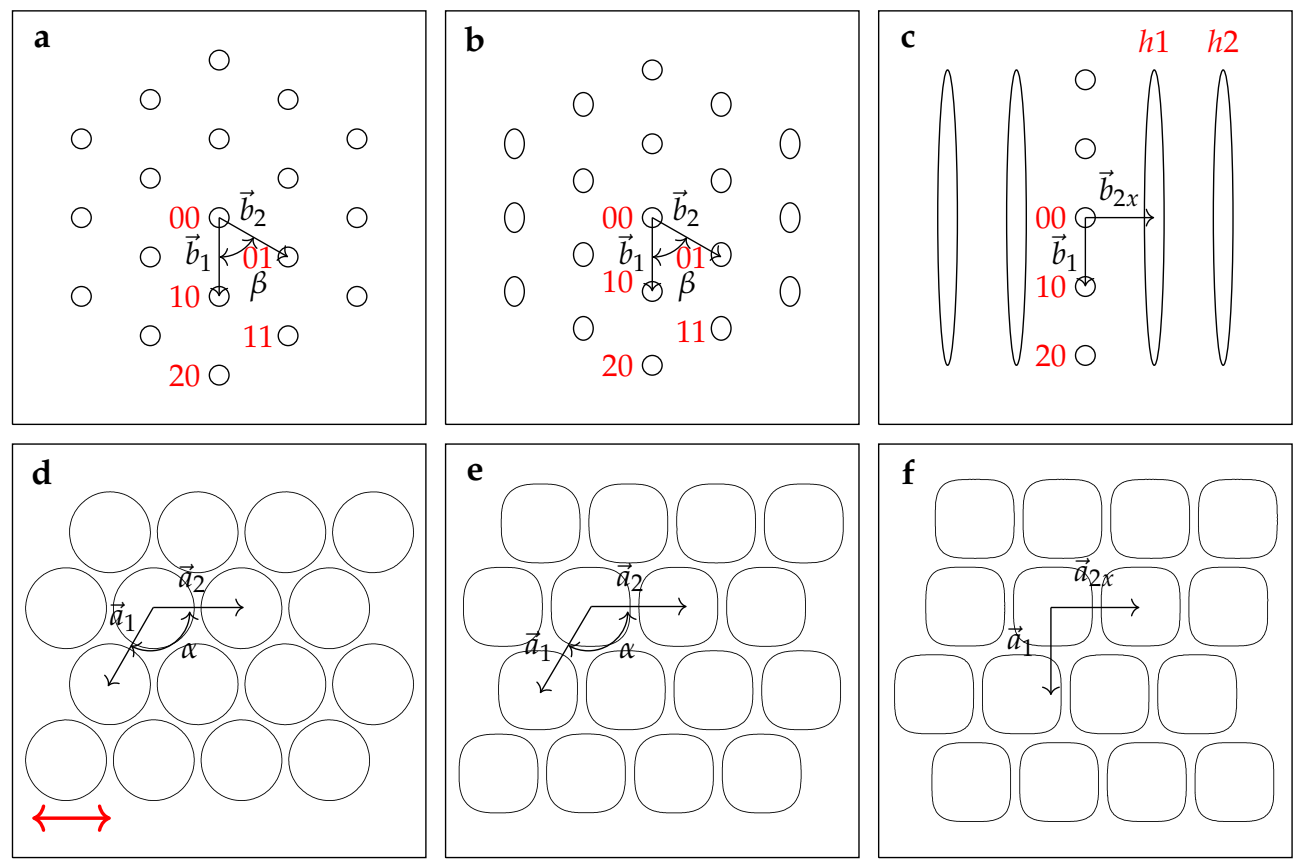

Figure 7. (a-c) The deduced structure factor $S(\vec{q})$ in reciprocal space and $(\mathbf{d}-\mathbf{f})$ the corresponding lattices in real space for the (a,d) solid spherical, $(\mathbf{b}, \mathbf{e})$ hollow semi-cubic and $(\mathbf{c}, \mathbf{f})$ hollow cube-like superballs. Here $\vec{b}_{1}$ and $\vec{b}_{2}$ are the primitive reciprocal basis vectors, separated by angle $\beta$, and $\vec{a}_{1}$ and $\vec{a}_{2}$, separated by angle $\alpha$, are the corresponding primitive lattice vectors. For the cube-like superballs we have instead defined $\vec{b}_{2 x}$ as the horizontal component that is perpendicular to $\vec{b}_{1}$. The Bragg peaks are labelled on their left. The direction of the mechanical rubbing is indicated in the images using a red arrow.

Thus, upon increasing the shape parameter $m$ of the superballs, several structural changes are observed in their lattice structures. First, the superballs have formed chains in the horizontal direction in the monolayers, which are aligned to the direction of the mechanical rubbing. Upon increasing the shape parameter $m$, the ratio between $a_{1}$ and $a_{2}$ increases, which indicates that the spacing between the chains increases, as expected for superballs [28]. This leads to the ability for the chains to slide along each other for the most cubic-like superballs. Second, the broadening in the $h 1$ and $h 2$ peaks can already be observed for the semi-cubic superballs, which indicates that their chains are able to slide more freely with respect to each other than the spherical superballs, showing that a continuous transition from the hexagonal lattice to a full sliding phase occurs. Clearly, in the superball monolayers, the order in the direction of the mechanical rubbing remains due to chain formation, but the order perpendicular to the direction of the mechanical rubbing is quickly lost as the chains can slide along each other. These observations confirm the lattice structures seen in the SEM images shown in Figure 1d-f.

\section{Conclusions}

Monolayers of differently shaped silica superballs, namely spherical, semi-cubic and cube-like, were prepared using the unidirectional rubbing method and analyzed with SAXS. 
In the 2D SAXS patterns some of the Bragg peaks appeared to be missing due to the presence of minima in the particle form factors. Due to the anisotropic shape and the hollow nature of the particles, it was difficult to predict which peaks are missing, which complicated the analysis of the patterns. By imaging the diffraction patterns of the monolayers over a range of rotations around the $x$-axis, the missing Bragg peaks were visualized. By doing so, the structural analysis was possible for all three superball shapes. We found that densely packed monolayer structures were obtained for all three types of particles using the unidirectional rubbing method. The spherical superballs formed hexagonal lattices with a high degree of positional order in all directions, whereas upon increasing the shape parameter $m$ it was observed that this high degree of positional order in the direction of rubbing remained, but weakened in the direction perpendicular to the direction of rubbing, resulting in the formation of the sliding phase for the cube-like superballs. In the future, we will use this analysis method to further study and characterize the lattices of colloidal superballs assembled using the unidirectional rubbing method.

Thus, by exploring the three-dimensional reciprocal space of a two-dimensional sample we were able to effectively exclude the influence of the anisotropic form factor and analyze the densely packed structures of the colloidal monolayers in greater detail. As a final remark, we note that the problems discussed in the present work are not X-ray specific, but can as well be of importance for small-angle neutron scattering studies. If samples possess periodic ordering in fewer dimensions, in 1D or 2D, a rotation of the sample may allow the revelation of all essential diffraction features even in the case of Bragg peaks coinciding with form factor minima.

Author Contributions: Conceptualization, D.N.t.N. and A.V.P.; methodology, D.N.t.N. and A.V.P.; software, D.N.t.N.; validation, D.N.t.N., J.-M.M. and A.V.P.; formal analysis, D.N.t.N.; investigation, D.N.t.N. and A.V.P.; writing-original draft preparation, D.N.t.N.; writing-review and editing, D.N.t.N., J.-M.M. and A.V.P.; visualization, D.N.t.N.; supervision, J.-M.M. and A.V.P.; project administration, A.V.P.; funding acquisition, A.V.P. All authors have read and agreed to the published version of the manuscript.

Funding: This research was funded by NWO grant number 712.015.003 (D.N.t.N), 016.Veni.192.119 (J.-M.M.) and 195.068.1113 (ESRF beamtime).

Institutional Review Board Statement: Not applicable.

Informed Consent Statement: Not applicable.

Data Availability Statement: The data presented in the study are available on request from the corresponding author.

Acknowledgments: Dominique Thies-Weesie is thanked for her help with the preparation of the spherical silica colloids. Nicholas Orr is thanked for his help at the beamline of the ESRF. Michela Brunelli and Daniel Hermida Merino are thanked for providing technical support at the beamline. Cedric Gommes is thanked for useful discussions. The Nederlandse Organisatie voor Wetenschappelijk Onderzoek (NWO) is thanked for providing the financial support.

Conflicts of Interest: The authors declare no conflict of interest.

\section{References}

1. Boles, M.A.; Engel, M.; Talapin, D.V. Self-assembly of colloidal nanocrystals: From intricate structures to functional materials. Chem. Rev. 2016, 116, 11220-11289. [CrossRef] [PubMed]

2. Xu, Z.; Wang, L.; Fang, F.; Fu, Y.; Yin, Z. A review on colloidal self-assembly and their applications. Curr. Nanosci. 2016, 12, 725-746. [CrossRef]

3. Chen, Q.; Bae, S.C.; Granick, S. Directed self-assembly of a colloidal kagome lattice. Nature 2011, 469, 381-384. [CrossRef] [PubMed]

4. Yan, J.; Han, M.; Zhang, J.; Xu, C.; Luijten, E.; Granick, S. Reconfiguring active particles by electrostatic imbalance. Nat. Mater. 2016, 15, 1095-1099. [CrossRef]

5. Zhang, J.; Grzybowski, B.A.; Granick, S. Janus particle synthesis, assembly, and application. Langmuir 2017, $33,6964-6977$. [CrossRef] 
6. Park, C.; Lee, T.; Xia, Y.; Shin, T.J.; Myoung, J.; Jeong, U. Quick, large-area assembly of a single-crystal monolayer of spherical particles by unidirectional rubbing. Adv. Mater. 2014, 26, 4633-4638. [CrossRef]

7. Park, C.; Koh, K.; Jeong, U. Structural Color Painting by Rubbing Particle Powder. Sci. Rep. 2015, 5, 1-5. [CrossRef]

8. Cademartiri, L.; Bishop, K.J.; Snyder, P.W.; Ozin, G.A. Using shape for self-assembly. Philos. Trans. R. Soc. A Math. Phys. Eng. Sci. 2012, 370, 2824-2847. [CrossRef]

9. Rossi, L.; Sacanna, S.; Irvine, W.T.; Chaikin, P.M.; Pine, D.J.; Philipse, A.P. Cubic crystals from cubic colloids. Soft Matter 2011, 7 , 4139-4142. [CrossRef]

10. Meijer, J.M.; Byelov, D.V.; Rossi, L.; Snigirev, A.; Snigireva, I.; Philipse, A.P.; Petukhov, A.V. Self-assembly of colloidal hematite cubes: A microradian X-ray diffraction exploration of sedimentary crystals. Soft Matter 2013, 9, 10729-10738. [CrossRef]

11. Meijer, J.M.; Pal, A.; Ouhajji, S.; Lekkerkerker, H.N.; Philipse, A.P.; Petukhov, A.V. Observation of solid-solid transitions in 3D crystals of colloidal superballs. Nat. Commun. 2017, 8, 1-8. [CrossRef]

12. Meijer, J.M.; Meester, V.; Hagemans, F.; Lekkerkerker, H.N.; Philipse, A.P.; Petukhov, A.V. Convectively assembled monolayers of colloidal cubes: Evidence of optimal packings. Langmuir 2019, 35, 4946-4955. [CrossRef]

13. Meijer, J.M.; Hagemans, F.; Rossi, L.; Byelov, D.V.; Castillo, S.I.; Snigirev, A.; Snigireva, I.; Philipse, A.P.; Petukhov, A.V. Selfassembly of colloidal cubes via vertical deposition. Langmuir 2012, 28, 7631-7638. [CrossRef]

14. Rossi, L.; Soni, V.; Ashton, D.J.; Pine, D.J.; Philipse, A.P.; Chaikin, P.M.; Dijkstra, M.; Sacanna, S.; Irvine, W.T. Shape-sensitive crystallization in colloidal superball fluids. Proc. Natl. Acad. Sci. USA 2015, 112, 5286-5290. [CrossRef]

15. Bartlett, P.; Ottewill, R.H. A neutron scattering study of the structure of a bimodal colloidal crystal. J. Chem. Phys. 1992, 96, 3306-3318. [CrossRef]

16. Vos, W.L.; Megens, M.; Van Kats, C.M.; Bösecke, P. X-ray diffraction of photonic colloidal single crystals. Langmuir 1997, 13, 6004-6008. [CrossRef]

17. Scotti, A.; Gasser, U.; Herman, E.S.; Pelaez-Fernandez, M.; Han, J.; Menzel, A.; Lyon, L.A.; Fernández-Nieves, A. The role of ions in the self-healing behavior of soft particle suspensions. Proc. Natl. Acad. Sci. USA 2016, 113, 5576-5581. [CrossRef]

18. Otsuki, A.; de Campo, L.; Garvey, C.; Rehm, C. H2O/D2O Contrast Variation for Ultra-Small-Angle Neutron Scattering to Minimize Multiple Scattering Effects of Colloidal Particle Suspensions. Colloids Interfaces 2018, 2, 37.10.3390/colloids2030037. [CrossRef]

19. Klokkenburg, M.; Erné, B.H.; Wiedenmann, A.; Petukhov, A.V.; Philipse, A.P. Dipolar structures in magnetite ferrofluids studied with small-angle neutron scattering with and without applied magnetic field. Phys. Rev. E Stat. Nonlinear Soft Matter Phys. 2007, 75, 1-9. [CrossRef]

20. Krouglov, T.; Bouwman, W.G.; Plomp, J.; Rekveldt, M.T.; Vroege, G.J.; Petukhov, A.V.; Thies-Weesie, D.M. Structural transitions of hard-sphere colloids studied by spin-echo small-angle neutron scattering. J. Appl. Crystallogr. 2003, 36, 1417-1423. [CrossRef]

21. Holderer, O.; Zolnierczuk, P.; Pasini, S.; Stingaciu, L.; Monkenbusch, M. A better view through new glasses: Developments at the Jülich neutron spin echo spectrometers. Phys. B Condens. Matter 2019, 562, 9-12. [CrossRef]

22. Gommes, C.J.; Reiner, Z.; Jaksch, S.; Frielinghaus, H.; Holderer, O. Inelastic Neutron Scattering Analysis with Time-Dependent Gaussian-Field Models. 2021, to be published.

23. Riekel, C.; Burghammer, M.; Davies, R. Progress in micro- and nano-diffraction at the ESRF ID13 beamline. IOP Conf. Ser. Mater. Sci. Eng. 2010, 14, 012013. [CrossRef]

24. Petukhov, A.V.; Meijer, J.M.; Vroege, G.J. Particle shape effects in colloidal crystals and colloidal liquid crystals: Small-angle X-ray scattering studies with microradian resolution. Curr. Opin. Colloid Interface Sci. 2015, 20, 272-281. [CrossRef]

25. Stöber, W.; Fink, A.; Bohn, E. Controlled growth of monodisperse silica spheres in the micron size range. J. Colloid Interface Sci. 1968, 26, 62-69. [CrossRef]

26. Sugimoto, T.; Sakata, T. Preparation of monodisperse pseudocubic $\alpha-\mathrm{Fe}_{2} \mathrm{O}_{3}$ particles from condensed ferric hydroxide gel. J. Colloid Interface Sci. 1992, 152, 587-590. [CrossRef]

27. Snigirev, A.; Kohn, V.; Snigireva, I.; Lengeler, B. A compound refractive lens for focusing high-energy X-rays. Nature 1996, 384, 49-51. [CrossRef]

28. Jiao, Y.; Stillinger, F.H.; Torquato, S. Optimal packings of superdisks and the role of symmetry. Phys. Rev. Lett. 2008, 100, 2-5. [CrossRef] 